\title{
Determinants of plasma cholesterol responsiveness to diet
}

\author{
BY MARGARET M. COBB AND HOWARD TEITLEBAUM \\ Laboratory of Biochemical Genetics and Metabolism, The Rockefeller University, \\ 1230 York Avenue, New York, New York 10021-6399, USA
}

(Received 6 February 1992 - Revised 16 February 1993 - Accepted 1 April 1993)

\begin{abstract}
Plasma cholesterol change, or 'responsiveness', to dietary saturated fat modification has long been acknowledged. The present study sought to determine the specific, predicted response of each cholesterol subfraction to known dietary manipulations. Two metabolically controlled diets, one with a low polyunsaturated:saturated fat (low $P: S$ ) ratio, and one with a high $P: S$ ratio were fed in a crossover design to sixty-seven normolipidaemic subjects pooled from six foregoing metabolic studies. A series of statistical analyses was performed to identify the lipids and subfractions independently affected by the diet crossover. Multivariate analysis of variance revealed that the changes in total cholesterol $(\Delta T C)$, low-density-lipoprotein-cholesterol $(\Delta \mathrm{LDL}-\mathrm{C})$, and high-density-lipoprotein-cholesterol ( $\triangle \mathrm{HDL}-\mathrm{C})$ were the only statistically significant diet-specific 'responsive' lipids. Multiple regression was performed to identify the independent predictors of $\triangle T C, \Delta L D L-C$ and $\triangle H D L-C$. It was found that age (years), extent of change in dietary saturated fat, and baseline LDL-C (mg/l) levels determine LDL-C change, while extent of change in saturated and polyunsaturated fat, and baseline HDL-C (mg/l) levels can predict HDL-C change. A series of equations to predict lipoprotein responsiveness to diet are derived for potential use in clinical practice.
\end{abstract}

Plasma cholesterol: Dietary fat: Lipoproteins: Man

Numerous studies have focused on the factors that regulate total cholesterol (TC) levels and can exert a beneficial change in lipid concentrations (Castelli et al. 1986; Lerner \& Kanner, 1986; Martin et al. 1986; Nutrition Committee, American Heart Association, 1988). By far the most intensely studied of these influences has been dietary, and more specifically the change in dietary saturated fatty acids (SFA). Several investigators have studied the extent of change, or 'responsiveness', as seen by a predictable change in total cholesterol ( $\triangle \mathrm{TC}$ ), to a change in dietary fat (Ahrens et al. 1957; Keys et al. 1957, 1965a, b; Grande \& Anderson, 1964; Hegsted et al. 1965; Keys \& Parlin, 1965; Jacobs et al. 1983).

The earliest ground-breaking work in the area of cholesterol responsiveness to dietary change was done by Keys et al. $(1957,1965 a, b)$. In 1965, following a study conducted under metabolic conditions, this group proposed an interrelationship between a change in dietary fatty acid composition and a subsequent $\triangle \mathrm{TC}$, which was later validated (Keys $e t$ al. 1965a, b). However, these researchers (Keys et al. 1965a; Jacobs et al. 1983) have only focused on the $\triangle \mathrm{TC}$. Although change in plasma TC manifests mainly in the low-densitylipoprotein subfraction (LDL-C), a change in dietary fatty acids seems to affect the highdensity-lipoprotein (HDL-C) fraction as well (Ehnholm et al. 1984; Kay et al. 1984; Sacks et al. 1986). To date no attempts have been made to predict specific changes in these lipoprotein subfractions with diet.

In a study by Grundy \& Vega (1988) subjects fed on metabolically-controlled formula diets with markedly contrasting polyunsaturated:saturated $(P: S)$ values showed a wide variability in $\mathrm{HDL}-\mathrm{C}$ response change $(\triangle \mathrm{HDL}-\mathrm{C})$ with diet in subjects with higher baseline 
levels, being more responsive to dietary change, i.e. there was a greater change in cholesterol for relatively lower change in dietary fat. Studies from the same laboratory (Grundy et al. 1986; Grundy, 1989) have shown that monounsaturated fatty acids (MUFA) are as effective as polyunsaturated fatty acids (PUFA) in lowering LDL-C and that the former do not appear to have the potential to lower HDL-C. Thus, both baseline HDL-C levels and the type of fatty acid replacement may be predictive of HDL-C lowering. We therefore pooled data from six similar studies (Fisher et al. 1983; Wissel et al. 1987; Zanni et al. 1987; Denke \& Breslow, 1988; Weintraub et al. 1988; Brinton et al. 1990) to describe the differential responsiveness to strict, metabolically controlled diets of the major cholesterol variables, TC, LDL-C and HDL-C.

\section{MATERIALS AND METHODS}

The present analysis is a composite of six previous studies (Table 1) conducted at two research institutions, The Rockefeller University in New York City and Harvard Medical School, Boston, Massachusetts. Research for all six studies was linked under the direction of a single senior investigator, Dr Jan Breslow (Fisher et al. 1983; Wissel et al. 1987; Zanni et al. 1987; Denke \& Breslow 1988; Weintraub et al. 1988; Brinton et al. 1990). The key common denominators of the six studies were metabolically controlled dietary change, identical biochemical methods, and similar study populations and research design permitting linkage for pooled analysis. For clarity of presentation, crossover is described as a change from a low $P: S$ to high $P: S$ regimen, as this is the regimen prescribed in practice.

\section{Study subjects}

Studies providing the database for this analysis spanned 9 years (1980-8). Before data analysis, predetermined selection criteria were developed: subjects had to be in excellent health and older than 18 years with lipoprotein profiles between the 25th and 75th percentile rank according to Lipid Research Clinics standards (Rifkind, 1989). Volunteers were recruited from staff and undergraduate work-study students from the two study institutions. Normocholesterolaemic patients who had entered the lipid clinic of each University for routine evaluation were also included.

The initial pooled subject group comprised seventy normolipidaemic participants; one subject was dropped from the analysis after developing severe hypertriglyceridaemia and two subjects (Weintraub et al. 1988) failed to complete the study diet protocol. Thus, a final pool of sixty-seven subjects participated in the six metabolic studies to completion and comprised the database for this analysis. Informed consent was obtained from all volunteers after review of the study protocols at each of the two locations.

Table 2 shows the selected clinical characteristics of age, body mass index $\left(\mathrm{kg} / \mathrm{m}^{2} ; \mathrm{BMI}\right)$, energy intakes, baseline (ad lib.) total lipids, cholesterol subfractions and ratios of all study subjects. This group comprised thirty-four men and thirty-three women with an average age of 25 years. The average BMI was $23 \mathrm{~kg} / \mathrm{m}^{2}$; an average energy intake $(\mathrm{kJ})$ was estimated for each subject to ensure weight maintenance. During the baseline period, females exhibited higher HDL-C than males $(P<0 \cdot 05)$.

\section{Diet protocols}

A low $P: S$ diet $(P: S \leqslant 0.6)$ was designed to exaggerate a typical diet rich in SFA and cholesterol, while a high $\mathrm{P}: \mathrm{S}$ diet $(\mathrm{P}: \mathrm{S}>1 \cdot 0)$ was tailored to simulate a more therapeutic diet rich in PUFA, low in SFA and considerably lower in dietary cholesterol. At both institutions, two such diets were administered to all subjects with an intervening 2-week 


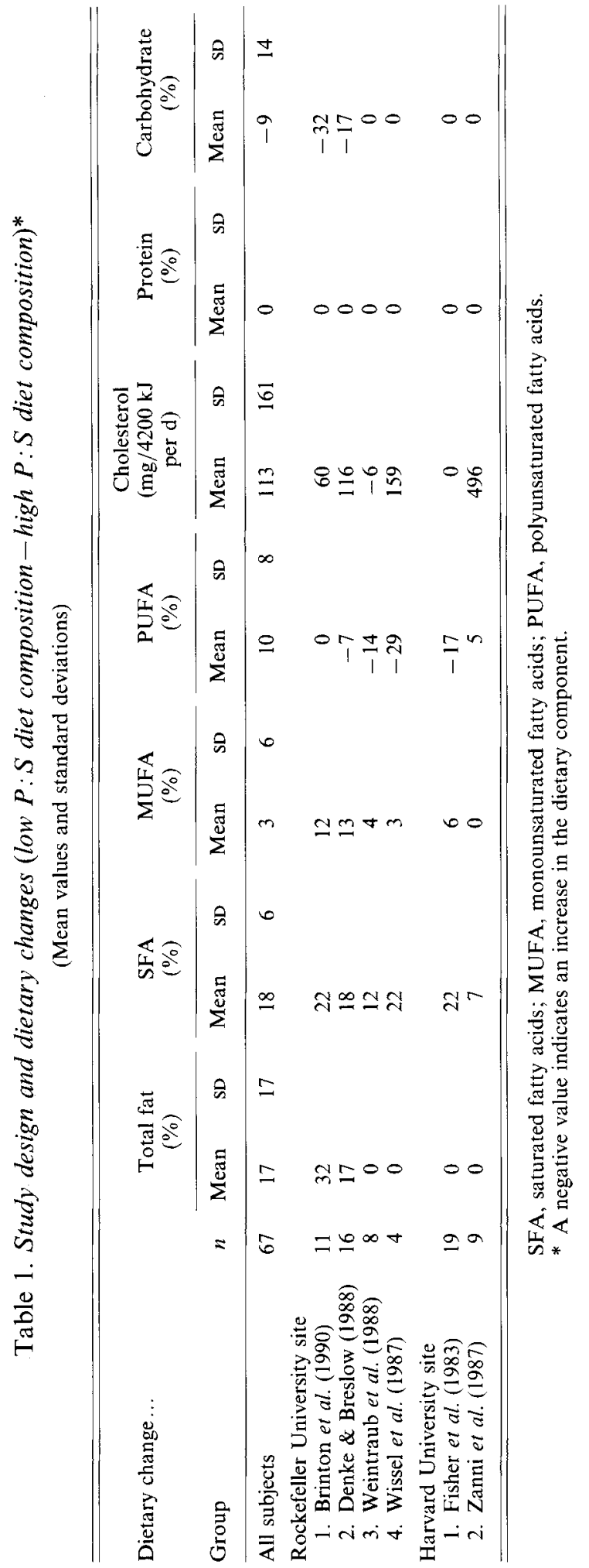




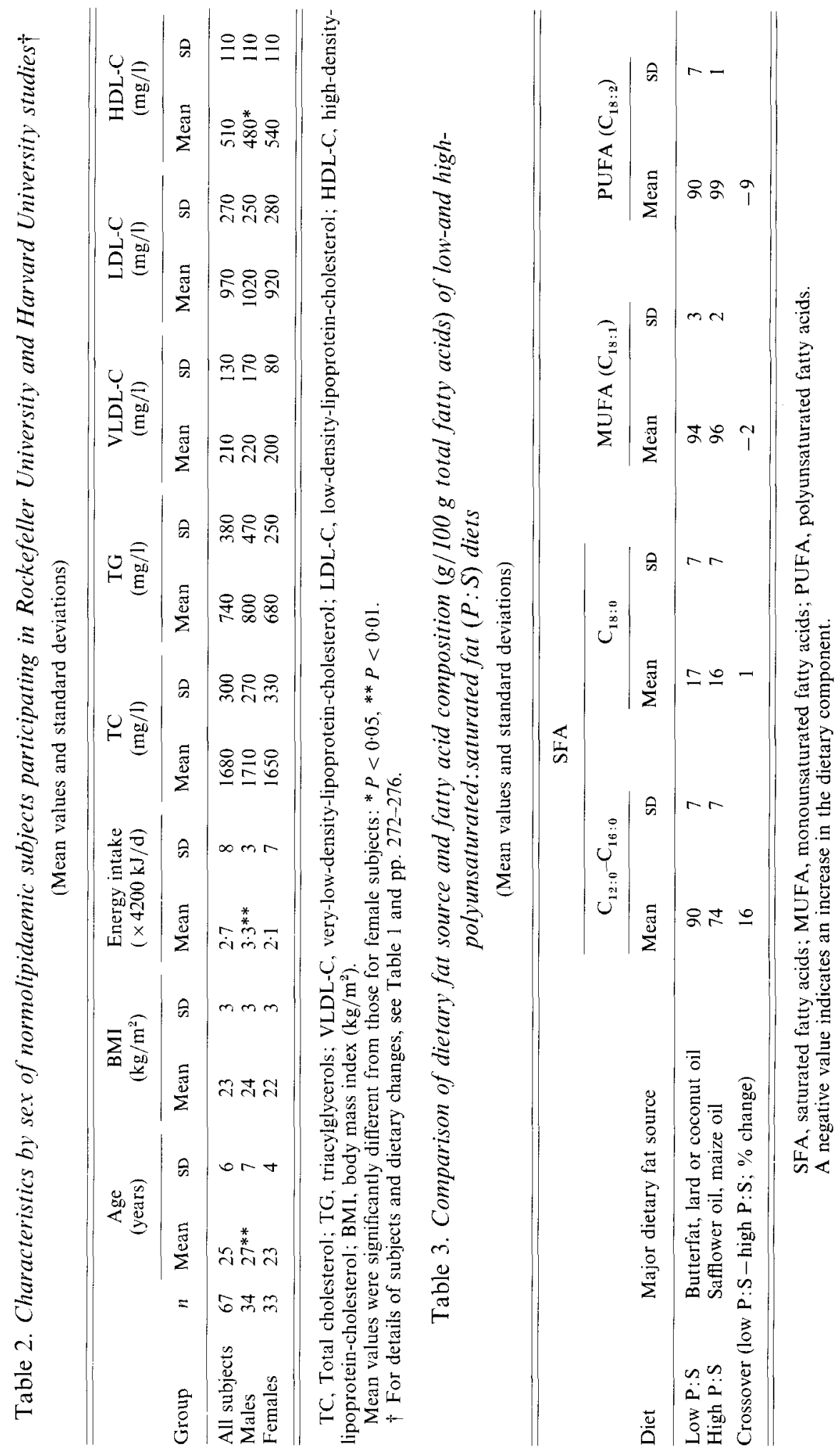


washout in each study. One diet with a low $\mathrm{P}: \mathrm{S}$ ratio or one with a high $\mathrm{P}: \mathrm{S}$ ratio were fed to each patient in a randomized order. A washout period separated each metabolic diet, so that each subject was given either a high-P:S diet followed by washout followed by low-P:S diet, or used a low-P:S diet first followed by a high-P:S diet with an intervening washout. Subjects were required to consume all food at each meal and were instructed to maintain their usual levels of physical activity throughout all phases of the study. To adjust for differences in energy intake across studies, the percentage of energy derived from each nutrient was calculated, and percentage change $(\% \Delta)$ calculated by difference.

The $\% \Delta$ for diet composition for each study are shown in Table 1 and fatty acid compositions are presented in Table 3 and in the published studies (Fisher et al. 1983; Wissel et al. 1987; Zanni et al. 1987; Denke \& Breslow, 1988; Weintraub et al. 1988; Brinton et al. 1990). Three of the six diet studies maintained the same total fat content at $310 \mathrm{~g} / \mathrm{kg}$ (Fisher et al. 1983; Zanni et al. 1987) or $420 \mathrm{~g} / \mathrm{kg}$ (Weintraub et al. 1988) following the diet crossover. The three remaining studies (Wissel et al. 1987; Denke \& Breslow, 1988; Brinton et al. 1990) reduced the total fat content. All studies showed a reduction in energy intake derived from SFA and a mean percentage decrease in total energy intake of $18 \%$. Except in the study carried out by Zanni et al. (1987), all dietary studies decreased the amount of MUFA by 3-12\%, with a mean decline of $3 \%$. All diet studies either maintained or increased the proportion of total energy derived from PUFA by $0-29 \%$, with a mean increase of $10 \%$. The diet crossover (Table 3 ) reduced $C_{12: 0}-C_{16: 0}$ chain lengths by $16 \%$ and stearate $\left(\mathrm{C}_{18: 0}\right)$ by an additional $1 \%$. Oleic acid $\left(\mathrm{C}_{18: 1}\right)$ and linoleic acid $\left(C_{18: 2}\right)$ concentrations of the diets were increased by 2 and $9 \%$ respectively. Dietary cholesterol was reduced during the high-P:S phase compared with low-P:S diet levels. Two exceptions were the formula diet study of Fisher et al. (1983), where there was no added cholesterol, and the study by Weintraub et al. (1988), which approximated dietary cholesterol intakes across both diet periods.

Data were adjusted for differences in diet and clinical characteristics in the present study.

\section{Analysis}

A minimum of three blood samples were collected at the end of each diet period and averaged, at both the Rockefeller and Harvard sites, and plasma quantified for total lipids and cholesterol subfractions by the same methods and standardized at the Center for Disease Control, Atlanta, Georgia. TC and triacylglycerol (TG) levels were assayed enzymically utilizing reagents from Boehringer Mannheim Biochemicals, Indianapolis, IN, USA. The HDL-C level was quantified as previously described (Warnick et al. 1982). The HDL-C plus LDL-C concentrations were determined after ultra centrifugation to separate the VLDL-C subfraction. LDL-C and VLDL-C levels were determined by difference as reported previously by Denke \& Breslow (1988).

As current guidelines recommend changes in absolute amount of lipids (mg/1), the change in lipid levels, calculated as the difference between the average lipid concentration measured during the low-P:S diet and the corresponding repeated lipid level quantified following the high-P:S diet, was derived individually for each subject. From these values dependent variables (i.e. 'diet-responsive' lipids) were calculated: $\Delta \mathrm{TC}, \Delta \mathrm{TG}, \Delta \mathrm{VLDL}-\mathrm{C}$, $\triangle \mathrm{LDL}-\mathrm{C}$, and $\triangle \mathrm{HDL}-\mathrm{C}$. All statistical analyses were calculated using Biomedical Computer Programs statistical software on a VAX 780 mainframe computer (BMDP: 1d, 2r, 4v, 8d and 6r; BMDP Software, Carey, NC, USA).

To identify the specific diet-responsive lipids, a multivariate analysis of covariance (MANCOVA) was performed with age, baseline lipoprotein profiles, group differences in diet (i.e. total fat, fatty acid composition, and cholesterol), diet-type (formula $v$. solid-food 
diets) and study site (Rockefeller $v$. Harvard University). Partial correlations and multivariate regression analyses (BMDP 6r; BMDP Software) were conducted to isolate the potential predictors of the identified diet-responsive variables $(\triangle \mathrm{TC}, \triangle \mathrm{LDL}-\mathrm{C}, \Delta \mathrm{HDL}$ C). By univariate analysis, the independent clinical variables included the following: age (years), sex, BMI, energy intake $(\mathrm{kJ} / \mathrm{d})$, and diet variables expressed as $\% \Delta$ following the diet crossover. The independent dietary composition variables including total nutrient compositions ( $\% \Delta$ total fat, $\% \Delta$ protein, and $\% \Delta$ carbohydrate), fatty acids compositions ( $\% \Delta$ SFA, $\% \Delta$ PUFA and $\% \Delta$ MUFA), and dietary cholesterol, expressed as square root of difference between the two metabolic diets and their ability to predict lipid changes, were studied. Baseline TC, LDL-C and HDL-C concentrations obtained before the metabolic studies were entered into the final model. All independent variables in the final regression model had coefficients significantly different from zero $(P<0 \cdot 05)$. For ease of interpretation the initial equation will be modified in a beneficial direction for each variable, that is to say a drop in LDL-C from the low- to high-P:S diet is seen as an improvement. The correlation between the estimated $\Delta \mathrm{TC}$ computed from the Minnesota equation, derived by Keys et al. $(1957,1959,1965 a, b)$, and using our derived model was calculated using BMDP2r (BMDP Software).

\section{RESULTS}

$\Delta \mathrm{TC}, \triangle \mathrm{LDL}-\mathrm{C}$ and $\triangle \mathrm{HDL}-\mathrm{C}$ pooled across all metabolic diet studies and by individual groups are presented in Table 4, with the MANCOVA results also shown. Values for $\Delta \mathrm{TG}$ and $\triangle$ VLDL-C were not significant and, thus, are not shown. The effect of the diet crossover was statistically significant $(P<0.001)$ in predicting these diet-responsive lipids. Across and within the six study groups a wide range of lipid responsiveness was observed in the total lipid changes and cholesterol subfraction differences. For all subjects combined, crossover from a low- to a high-P: $S$ diet resulted in an average reduction in TC of $380 \mathrm{mg} / 1$ with associated decreases in all subfractions. LDL-C and HDL-C subfractions showed decreases of 280 and $80 \mathrm{mg} / 1$ respectively following the dietary crossover. These changes were reflected in LDL-C ranging from 120 to $650 \mathrm{mg} / \mathrm{l}$ for the Brinton et al. (1990) and Wissel et al. (1987) groups respectively. Even more dramatic, the change in HDL-C showed a 12-fold difference across study groups. Weintraub et al. (1988) showed a $10 \mathrm{mg} / 1$ increase in HDL-C, whereas the Brinton et al. (1990) study subjects showed HDL-C decreased by an average of $120 \mathrm{mg} / \mathrm{l}$. This variability was used to advantage in isolating the predictors of lipoprotein responsiveness.

A profile of independent variables (clinical, dietary and baseline lipids) was pooled across all studies and the univariate relationship was determined.

\section{Change in $T C$ and $L D L-C$}

The univariate correlates for $\triangle \mathrm{TC}$ and $\triangle \mathrm{LDL}-\mathrm{C}$ revealed that age (years) and the baseline TC and LDL-C levels were the significant univariate clinical and baseline lipid predictors of the changes. Among the metabolic diet variables, the decrease in SFA and increase in PUFA were correlated with both $\triangle \mathrm{TC}$ and $\triangle \mathrm{LDL}-\mathrm{C}$.

\section{Change in $H D L-C$}

The univariate correlates for $\triangle$ HDL-C were sex, baseline VLDL-C level, and HDL-C concentration (Table 5). All dietary change variables, except protein, were correlated with $\triangle H D L-C$. Among the metabolic diet variables, a decrease in SFA and MUFA, or dietary cholesterol resulted in greater lowering of HDL-C. Conversely, an increase in dietary carbohydrate and PUFA promoted HDL-C lowering. 
Table 4. The effect of changing from a low-polyunsaturated: saturated fat $(P: S)$ diet to a high-P:S diet on diet responsive lipids in normolipidaemic subjects participating in the Rockefeller University and Harvard University studies*

(Mean values and standard deviations)

\begin{tabular}{|c|c|c|c|c|c|c|}
\hline \multirow[t]{2}{*}{ Cholesterol and subfraction response $(\mathrm{mg} / \mathrm{l}) \ldots$} & \multicolumn{2}{|c|}{$\Delta \mathrm{TC}$} & \multicolumn{2}{|c|}{$\Delta \mathrm{LDL}-\mathrm{C}$} & \multicolumn{2}{|c|}{$\Delta \mathrm{HDL}-\mathrm{C}$} \\
\hline & Mean & SD & Mean & SD & Mean & SD \\
\hline All subjects & 380 & 120 & 280 & 110 & 80 & 50 \\
\hline $\begin{array}{l}\text { Rockefeller University Study } \\
\text { 1. Brinton et at. (1990) } \\
\text { 2. Denke \& Breslow (1988) } \\
\text { 3. Weintraub et al. (1988) } \\
\text { 4. Wissel et al. (1987) }\end{array}$ & $\begin{array}{l}430 \\
320 \\
280 \\
730\end{array}$ & $\begin{array}{l}50 \\
50 \\
60 \\
90\end{array}$ & $\begin{array}{l}260 \\
230 \\
180 \\
450\end{array}$ & $\begin{array}{r}80 \\
70 \\
30 \\
160\end{array}$ & $\begin{array}{r}120 \\
80 \\
-10 \\
110\end{array}$ & $\begin{array}{l}60 \\
20 \\
30 \\
70\end{array}$ \\
\hline $\begin{array}{l}\text { Harvard University Study } \\
\text { 1. Fisher et al. (1983) } \\
\text { 2. Zanni et al. (1987) }\end{array}$ & $\begin{array}{l}410 \\
310\end{array}$ & $\begin{array}{l}60 \\
40\end{array}$ & $\begin{array}{l}320 \\
290\end{array}$ & $\begin{array}{r}120 \\
50\end{array}$ & $\begin{array}{l}80 \\
90\end{array}$ & $\begin{array}{l}20 \\
10\end{array}$ \\
\hline
\end{tabular}

$\Delta \mathrm{TC}$, change in total cholesterol; $\triangle \mathrm{LDL}-\mathrm{C}$, change in low-density-lipoprotein-cholesterol; $\Delta \mathrm{HDL}-\mathrm{C}$, change in high-density-lipoprotein-cholesterol.

Mean values were analysed by multivariate analysis of covariance (MANCOVA): overall diet effect, $P<0.0001 ; \Delta \mathrm{TC}, P<0.03 ; \Delta \mathrm{LDL}, P<0.05 ; \Delta \mathrm{HDL}, P<0.0005$.

* For details of subjects and dietary changes, see Table 1 and pp. 272-276.

\section{Change in $T C$}

The best predictor of $\triangle \mathrm{TC}$ was a two-variable model $(P<0 \cdot 001)$ :

$$
\begin{aligned}
& \Delta \mathrm{TC}(\mathrm{mg} / \mathrm{l})=0.79(\text { age } ; \text { years })+1.03(\% \Delta \mathrm{SFA}) \\
& \text { (SE 0.12) (SE 0.20) }
\end{aligned}
$$

accounting for $85 \%$ of the variance. Inspection of the standardized regression coefficient $(0 \cdot 47-0.48)$ showed a similar contribution of these two variables to prediction of the $\triangle T C$. There was no improvement in the adjusted $R^{2}$ change with addition of a third variable to this model. The Minnesota equation (Keys et al. 1957, 1965a, b; Hegsted et al. 1965) when applied to our results explained $82 \%$ of the variance in $\triangle T C$.

\section{Change in $L D L-C$}

The best model for $\triangle \mathrm{LDL}-\mathrm{C}$ was a three-variable model $(P<0.001)$ :

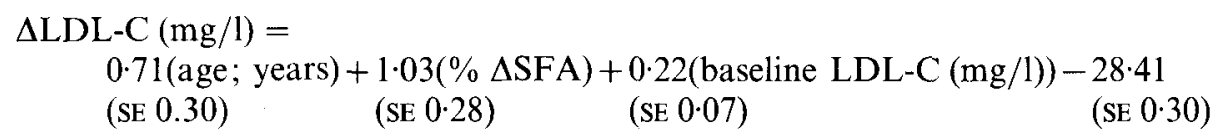

This model accounted for $36 \%$ of the variance in $\triangle \mathrm{LDL}-\mathrm{C}$, with similar contributions by all variables to model prediction.

\section{Change in $H D L-C$}

The best three-variable model $(P<0.001)$ included dietary SFA and PUFA, plus the baseline HDL-C level. The multiple-regression equation was:

$$
\begin{aligned}
& \Delta \mathrm{HDL}-\mathrm{C}(\mathrm{mg} / \mathrm{l})=0 \cdot 31(\% \Delta \mathrm{SFA})-0 \cdot 40(\% \Delta \mathrm{PUFA})+0 \cdot 13(\text { baseline HDL-C }(\mathrm{mg} / \mathrm{l})) \\
& \text { (SE 0.12) } \\
& \text { (SE 0.10) } \\
& \text { (SE 0.04) }
\end{aligned}
$$

and accounted for a predictive variance of $74 \%$. Sex was a significant predictor $(P<0 \cdot 01)$, 
Table 5. Univariate determinants of dietary responsiveness to low-polyunsaturated: saturated fat $(P: S)$ and high $P: S$ diets in sixty-seven normolipidaemic subjects participating in the Rockefeller University and Harvard University studies $\uparrow$

\begin{tabular}{|c|c|c|c|}
\hline & \multirow{2}{*}{$\begin{array}{c}\text { Total lipids } \\
\Delta \mathrm{TC}\end{array}$} & \multicolumn{2}{|c|}{ Cholesterol subtractions } \\
\hline & & $\triangle \mathrm{LDL}-\mathrm{C}$ & $\triangle \mathrm{HDL}-\mathrm{C}$ \\
\hline \multicolumn{4}{|l|}{ Clinical variables } \\
\hline Age (years) & $0.335^{* * *}$ & $0 \cdot 357^{* *}$ & $-0 \cdot 129$ \\
\hline $\operatorname{Sex}(M, F 0,1)$ & -0.240 & $-0 \cdot 216$ & $0.261^{*}$ \\
\hline $\operatorname{BMI}\left(\mathrm{kg} / \mathrm{m}^{2}\right)$ & 0.029 & 0.066 & $-0 \cdot 121$ \\
\hline Energy intake $(\mathrm{kJ} / \mathrm{d})$ & 0.232 & 0.188 & -0.080 \\
\hline \multicolumn{4}{|l|}{ Diet variables } \\
\hline \multicolumn{4}{|l|}{ Fatty acids composition } \\
\hline$\% \triangle \mathrm{SFA}$ & $0 \cdot 374 * *$ & $0.240^{*}$ & $0.233^{*}$ \\
\hline$\% \triangle \mathrm{PUFA}$ & $-0 \cdot 314^{* *}$ & $-0 \cdot 268 *$ & $0 \cdot 344^{* *}$ \\
\hline$\% \triangle \mathrm{MUFA}$ & -0.090 & 0.070 & $0 \cdot 247^{*}$ \\
\hline $\begin{array}{l}\text { Cholesterol (SQRT } \\
\left.\left(\mathrm{C}_{1}-\mathrm{C}_{2}\right)\right)\end{array}$ & -0.005 & $0 \cdot 121$ & $0.241^{*}$ \\
\hline Carbohydrates & 0.051 & 0.085 & $-0 \cdot 396^{* * *}$ \\
\hline Protein & 0.000 & $0 \cdot 000$ & 0.000 \\
\hline \multicolumn{4}{|l|}{ Baseline lipids (mg/l) } \\
\hline $\mathrm{TC}$ & $0.439 * * *$ & $0 \cdot 421^{* * *}$ & 0.001 \\
\hline $\mathrm{TG}$ & $0 \cdot 164$ & $0 \cdot 123$ & $-0 \cdot 127$ \\
\hline VLDL-C & $0 \cdot 220$ & $0 \cdot 096$ & $0.578 * * *$ \\
\hline LDL-C & $0.431^{* * *}$ & $0 \cdot 490^{* * *}$ & $0 \cdot 166$ \\
\hline HDL-C & -0.039 & $-0 \cdot 146$ & $0.460^{* * *}$ \\
\hline
\end{tabular}

$\Delta \mathrm{TC}$, change in total cholesterol; $\% \triangle \mathrm{SFA}$, percentage change in saturated fatty acids; $\% \Delta \mathrm{PUFA}$, percentage change in polyunsaturated fatty acids; $\% \triangle$ MUFA, percentage change in monounsaturated fatty acids; BMI, body mass index $\left(\mathrm{kg} / \mathrm{m}^{2}\right)$; SQRT $(\mathrm{Cl}-\mathrm{C} 2)$, square root difference in dietary cholesterol; TG, triacylglycerols; VLDL-C, very-low-density-lipoprotein-cholesterol; LDL-C, low-density-lipoprotein-cholesterol; HDL-C, highdensity-lipoprotein-cholesterol.

* $P<0.05 ; * * P<0.025 ; * * * P<0.01$.

$\dagger$ For details of subjects and dietary changes, see Table 1 and pp. 272-276.

in addition to $\% \triangle \mathrm{SFA}$ and $\% \triangle \mathrm{PUFA}$, but not greater than baseline HDL-C levels (values not presented).

\section{DISCUSSION}

We found that age (years), $\% \triangle \mathrm{SFA}$, and baseline LDL-C $(\mathrm{mg} / \mathrm{l})$ levels determine $\triangle \mathrm{TC}$ and $\Delta \mathrm{LDL}$, while $\% \triangle \mathrm{SFA}$ and $\% \triangle \mathrm{PUFA}$ and baseline HDL-C $(\mathrm{mg} / \mathrm{l})$ levels predict $\Delta \mathrm{HDL}-$ C. Each $10 \%$ reduction in SFA results in a decrease in LDL-C and HDL-C of approximately 100 and $30 \mathrm{mg} / \mathrm{l}$ respectively in an average member of the normolipidaemic population. The common term in both these equations is SFA, explaining the positive correlation between $\triangle$ LDL-C and $\triangle$ HDL-C. Although Keys (1984) proposed manipulation of only one dietary variable at a time, in clinical practice this is not feasible. In fact, the Nutrition Committee, American Heart Association (1988) recommends that 'all Americans from age 2 years and older consume a diet lower in total fat (to $30 \%$ ), decreased in SFA $(<10 \%)$, and less than $300 \mathrm{mg}$ cholesterol/d'. The National Cholesterol Education Program Expert Panel (1988) guidelines further recommend that PUFA should not exceed $10 \%$ of energy and MUFA should make up $10-15 \%$ of total energy.

The first algorithm (equation 1) shows that within this subject population $\Delta \mathrm{TC}$ is best 
predicted by age and a specific $\% \triangle$ SFA. Hypothetically, if a patient aged 20 years presents with a TC of $2100 \mathrm{mg} / \mathrm{l}$ and the physician wishes to effect a decrease in this concentration of $300 \mathrm{mg} / 1$ to a final level of $1800 \mathrm{mg} / 1$, the resultant necessary reduction in percentage of total energy intake from SFA is 13.8 . This patient, under rigorous guidance, would need to reduce his SFA intake from that of about $24 \%$ to a level of approximately $10 \%$ total energy from SFA. This reduction appears rather unrealistic with the patient's young age contributing to the necessity for such a severe dietary manipulation. For example, a patient aged 30 years who desired the same reduction in TC of $300 \mathrm{mg} / 1$ would require a drop in SFA intake of considerably less, namely a reduction of only $6 \cdot 1 \%$ total energy intake from 24 to $18 \%$ total energy intake from SFA. In agreement with our findings, others have shown suppression of LDL-receptor activity by SFA and ageing (Grundy et al. 1985; Spady \& Dietschy, 1985).

The previously described example demonstrates the utility of these equations and allows a health care professional to calculate the average $\% \Delta$ SFA modification to achieve a treatment goal. However, we would like to underscore that any estimate of the average amount of dietary change has a confidence interval about the mean and, therefore, must be interpreted within a range. These results, however, compared favourably with the earliest reported of such equations derived by others (Keys et al. 1957, 1965a, b; Hegsted et al. 1965). We found a highly significant correlation between our equation and the Minnesota equation (Keys et al. 1957, 1965a, b; Hegsted et al. 1965; $P<0.0001$ ). However, the predicted $\triangle \mathrm{TC}$ of the Minnesota equation tended to overestimate somewhat the observed changes in $\triangle \mathrm{TC}$ in our group, especially for the younger patients. This small overestimation is not surprising as it was derived from a data set including older male subjects, and may not be applicable to younger subjects. Our equation may be advantageous in clinical practice, since the patient's age is easily obtained, and estimation of a \% $\triangle P U F A$ would not be required. Thus, this equation may have special applicability in younger persons.

The crossover to a diet high in carbohydrate and low in total fat content has long been shown to lower plasma cholesterol levels (Keys et al. 1965a). However, dietary carbohydrates and oleic acid have been shown to produce a 'neutral' effect on serum cholesterol levels. In this analysis, neither the change in dietary carbohydrate nor total dietary fat were significant predictors of $\triangle \mathrm{TC}$ beyond age and dietary SFA content. This is not surprising as total fat and carbohydrate were maintained in four of the six protocols, whereas dietary SFA content was dramatically reduced.

More recent evidence suggests that all SFA are not equal in their ability to elevate plasma cholesterol concentrations (Cobb, 1992). Stearic acid $\left(\mathrm{C}_{18: 0}\right)$ appears to affect plasma cholesterol less than other SFA (Bonanome \& Grundy, 1988). Keys et al. (1965a) recommended that $\mathrm{C}_{18: 0}$ be subtracted from the dietary SFA content when calculating the plasma cholesterol change. In practice, however, $\mathrm{C}_{18: 0}$ is rarely removed when the equation is used to predict dietary responsiveness (Grundy \& Denke, 1990). In the present study, with mixed natural diets, subtraction of $\mathrm{C}_{18: 0}$ content did not significantly alter the predictive equations.

Our second algorithm (equation 2) indicates that a unit desired reduction in LDL-C is dependent on dietary $\% \triangle \mathrm{SFA}$, age and baseline LDL-C. Applying this equation, we assume a case patient aged 20 years has a baseline LDL-C of $1000 \mathrm{mg} / \mathrm{l}$, with the goal being to reduce this concentration by $300-700 \mathrm{mg} / 1$. Thus, this patient would require a change in dietary SFA of $21.6 \%$ total energy intake. Supposing a patient of similar age and desired drop in LDL-C has a higher baseline LDL-C of $1300 \mathrm{mg} / \mathrm{l}$, the required dietary $\% \triangle$ SFA would be a reduction of only 15.2 for this patient. This is a less severe requirement than the first patient of the same age and desired LDL-C reduction. The latter patient, 
requiring less change in dietary SFA, is more responsive to dietary manipulation. Grundy \& Vega (1988) have demonstrated a positive correlation between baseline LDL-C levels and $\triangle \mathrm{LDL}$ to diet.

The third algorithm (equation 3) predicts responsiveness for a given desired change (presumably, an increase in HDL-C). This algorithm can be solved for the individual subject only after equations 1 and 2 have been solved as $\% \triangle$ PUFA remains the only unknown variable in the algebraic solution. For example, taking the 20 -year-old male with a LDL-C of $1000 \mathrm{mg} / 1$ and the 30 -year-old male with a LDL-C of $1300 \mathrm{mg} / 1$ (equation 2), assume both have a baseline HDL-C level of $450 \mathrm{mg} / \mathrm{l}$ with a $\triangle H D L-C$ of zero, the near optimal case, the resultant required $\% \Delta$ total energy intake from PUFA would be a reduction of 31.3 and 21.5 respectively.

Given the current typical PUFA intake in Western societies of $6 \%$ total energy intake/d (National Center for Health Statistics, 1981), such a reduction in PUFA would be unrealistic. Adjustment to a more sensible clinical dimension could be achieved by substituting a reasonable $\triangle \mathrm{HDL}-\mathrm{C}$ or known change in $\% \triangle P U F A-f a t$. Since a $10 \%$ $\triangle \mathrm{PUFA}$ is reasonable, substituting this value into equation 3 for these same two hypothetical subjects yields a decrease in plasma HDL-C of approximately 90 and $40 \mathrm{mg} / 1$ for the younger and older patient respectively.

The fact that females generally present with higher baseline HDL-C than males (Albers et al. 1976) necessitates the input of typical female baseline HDL-C levels to determine the effect of sex on the responsiveness of HDL-C, as expressed in equation 3. Assuming our two hypothetical patients to be female, with baseline HDL-C levels of $600 \mathrm{mg} / \mathrm{l}$, and keeping all other known terms similar to those of the two male subjects described previously, a $10 \%$ increase in PUFA yields a HDL-C decrease of about 110 and $60 \mathrm{mg} / 1$ respectively for the younger and older females. Compared with the two male counterparts $(90$ and $40 \mathrm{mg}$ HDL-C/ 1 decrease), the decrease in HDL-C for the two females was greater and, theoretically, less desirable.

In the present study the change in MUFA was not a statistically important predictor of HDL-C decrease, compared with SFA and PUFA composition. Although some investigators (Grundy et al. 1986; Grundy, 1989), using formula diets containing very high amounts of MUFA ( $28 \%$ total energy intake), reported that replacement of SFA with MUFA prevents the $\triangle$ HDL-C, this cannot be interpreted from our study. More recent studies (Dreon et al. 1990), using solid food diets with SFA composition held constant, showed that both PUFA- and MUFA-fat replacement produced the same HDL-C changes in subjects after a diet-crossover. In our composite diet analysis SFA was not held constant and relatively small changes in MUFA were employed in most studies, thus, potentially limiting interpretation of the role of MUFA in predicting $\triangle \mathrm{HDL}$.

\section{Conclusions}

The findings of the present study have potential implications for epidemiological intervention in the normolipidaemic population. As illustrated in equations 2 and 3, the amount of change in LDL-C, and per unit change in HDL-C fraction in normolipidaemic subjects, is partially dictated by the dietary SFA content. Currently, the Nutrition Committee, American Heart Association (1988) recommends diet modification for all Americans. Broad-sweeping preventive approaches include reduction of $\mathrm{TC}$ levels in the general population $v$. a more-high-risk strategy targeted at subjects with elevated LDL-C levels. Results from the present study in normolipidaemic subjects support the latter approach. Younger subjects with lower LDL-C levels are not as diet responsive. Furthermore, young women with low LDL-C and high HDL-C may, in fact, not benefit from this dietary intervention. 
The authors extend appreciation to Dr Jan Breslow for supplying the original data in this manuscript. Gratitude is offered to Mr Michael Friedmann for his assistance in preparation of this manuscript. This work was supported in part by a General Clinical Research Center grant (RR00102) and general support from the Pew Trust at The Rockefeller University. In addition funds were generously donated by Suzanne and Irving Karpas, Karpas Health Information Center, Beth Israel Medical Center, New York, NY, and The Margolis Foundation.

\section{REFERENCES}

Ahrens, E. H. Jr, Hirsch, I. \& Insull, W. Ir (1957). The infuence of dietary fats on serum-lipid levels in man. Lancet i, 943-963.

Albers, J. J., Wahl, P. W., Cabana, V. G., Hazzard, W. R. \& Hoover, J. J. (1976). Quantitation of apolipoprotein AI of human plasma high density lipoprotein. Metabolism 25, 633-644.

Bonanome, A. \& Grundy, S. M. (1988). Effect of dietary stearic acid on plasma cholesterol and lipoprotein levels. New England Journal of Medicine 48, $1244-1248$.

Brinton, E. A., Eisenberg, S. \& Breslow, J. L. (1990). A low-fat diet decreases high density lipoprotein (HDL) cholesterol levels by decreasing HDL apolipoprotein transport rates. Joumal of Clinical Investigation 85 , $144-151$

Castelli, W. P., Garrison, R. J. \& Wilson, P. W. F. (1986). Incidence of coronary heart disease and lipoprotein levels: The Framingham Study. Journal of the American Medical Association 256, 2835-2838.

Cobb, T. (1992). Effects of dietary stearic acid on plasma cholesterol levels. Southern Medical Journal 85, 25-28.

Denke, M. A. \& Breslow, J. L. (1988). Effects of a low fat diet with and without intermittent saturated fat and cholesterol ingestion on plasma lipid, lipoprotein, and apolipoprotein levels in normal volunteers. Journal of Lipid Research 29, 963-969.

Dreon, D. M., Vranizan, K. M., Krauss, R. M., Austin, M. A.\& Wood, P. (1990). The effects of polyunsaturated fat on plasma lipoproteins. Journal of the American Medical Association 23, 2463-2466.

Ehnholm, C., Huttunen, J. K. \& Pietinen, P. (1984). Effect of a diet low in saturated fatty acids on plasma lipids, lipoproteins and HDL subfractions. Arteriosclerosis 4, 265-269.

Fisher, E. A., Blum, C. B., Zannis, V. I. \& Breslow, J. L. (1983). Independent effects of dietary saturated fat and cholesterol on plasma lipids, lipoproteins, and Apolipoprotein E. Journal of Lipid Research 24, $1039-1048$.

Grande, F. \& Anderson, J. T. (1964). Prediction of serum cholesterol changes caused dietary fat in man. Minnesota Medicine 47, 645-650.

Grundy, S. M. (1989). Comparison of monounsaturated fatty acids and carbohydrates for lowering plasma cholesterol. New England Journal of Medicine 314, 745-748.

Grundy, S. M. \& Denke, M. A. (1990). Dietary influences on serum lipids and lipoproteins. Journal of Lipid Research 31, 1149-1172.

Grundy, S. M., Nix, D., Whelan, M. F. \& Franklin, L. (1986). Comparison of three cholesterol-lowering diets in normolipidemic men. Journal of the American Medical Association 256, 2351-2355.

Grundy, S. M. \& Vega, G. L. (1988). Plasma cholesterol responsiveness to saturated fatty aids. American Joumal of Clinical Nutrition 47, 822-824.

Grundy, S. M., Vega, G. L.\& Bilheimer, D. W. (1985). Kinetic mechanisms determining variability in low density lipoprotein levels and rise with age. Arteriosclerosis 5, 623-630.

Hegsted, D. M., McGandy, R. B., Myers, M. L. \& Stare, F. J. (1965). Quantitative effects of dietary fat on serum cholesterol in man. American Journal of Clinical Nutrition 17, 281-295.

Jacobs, D. R. Jr, Anderson, J. T. \& Hannan, P. (1983). Variability in individual serum cholesterol response to change in diet. Arteriosclerosis 3, 349-356.

Kay, R. M., Jacobs, M., Katan, M. B., Miller, N. E. \& Lewis, B. (1984). Relationship between changes in plasma lipoprotein concentrations and faecal steroid excretion during consumption of four experimental diets. Atherosclerosis 55, 15-23.

Keys, A., Anderson, J. T. \& Grande, F. (1957). Prediction of serum cholesterol responses of man to changes in fats in the diet. Lancet ii, 959-966.

Keys, A., Anderson, J. T. \& Grande, F. (1959). Serum cholesterol in man: diet fat and intrinsic responsiveness. Circulation 19, 201-214.

Keys, A., Anderson, J. T. \& Grande, F. (1965a) Serum cholesterol response to changes in the diet. Iodine value of dietary fat versus 2S-P. Metabolism 14, 747-758.

Keys, A., Anderson, J. T. \& Grande, F. (1965b). Serum cholesterol response to changes in the diet. IV. Particular saturated fatty acids in the diet. Metabolism 14, 776-787.

Keys, A. K. (1984). Serum cholesterol response to dietary cholesterol. American Journal of Clinical Nutrition 40. 35I-359.

Keys, A. \& Parlin, R. W. (1965). Serum cholesterol response to changes in dietary lipids. American Journal of Clinical Nutrition 17, 175-181. 
Lerner, D. J. \& Kanner, W. B. (1986). Patterns of coronary heart disease morbidity and mortality in the sexes: a 26-year follow-up of the Framingham population. American Heart Joumal 111, 383-390.

Martin, M. J., Hulley, S. B., Browner, W. S., Kuller, L. H. \& Wentwort, D. (1986). Serum cholesterol, blood pressure and mortality: implications from a cohort of 361,662 men. Lancet ii, 9333-9936.

National Center for Health Statistics (1981). The Second National Health and Nutrition Examination Survey. Vital and Health Statistics Series 1, no. 15. DHHS Publication no. (PHS) 81-1317. Washington, DC: Government Printing Office.

National Cholesterol Education Program Expert Panel (1988). Report of the National Cholesterol Education Program Expert Panel on detection. evaluation and treatment of high blood cholesterol in adults. Archives of Internal Medicine 148, 36-69.

Nutrition Committee, American Heart Association (1988). Dietary guidelines for healthy American adults. A statement for physicians and health professionals by the Nutrition Committee, American Heart Association. Circulation 77, 721A-724A.

Rifkind, B. M. (editor) (1989). In The Lipid Research Clinies Populations Studies Data Book. vol. I, The Prevalence Study. NIH Publication no. 80-1527, pp. 70-73. Bethesda: NIH.

Sacks, F. M., Handysides, G. H. J., Mariais, G. E., Rosner, B. \& Kass, E. H. (1986). Effects of a low-fat diet on plasma lipoprotein levels. Archives of Internal Medicine 146, 1573-1577.

Spady, D. K. \& Dietschy, J. M. (1985). Dietary saturated triacylglycerols suppress hepatic low density lipoprotein receptor activity in the hamster. Proceedings of the National Academy of Sciences, USA 82, 4526-4530.

Warnick, G. R., Benderson, J. \& Albers, J. J. (1982). Dextran sulfate- $\mathrm{Mg}^{2+}$ precipitation procedure or quantitation of high-density-lipoprotein cholesterol. Clinical Chemistry 28, 1379-1388.

Weintraub, M. S., Zechner, R., Brown, A., Eisenberg, S. \& Breslow, J. L. (1988). Dietary polyunsaturated fats of the $\omega-6$ and $\omega-3$ series reduce postprandial lipoprotein levels. Chronic and acute effects of fat saturation on postprandial lipoprotein metabolism. Journal of Clinical Investigation 82, 1884-1893.

Wissel, P. S., Denke, M. A. \& Inturrisi, C. A. (1987). A comparison of the effects of a macrobiotic diet and a Western diet on drug metabolism and plasma lipids in man. European Journal of Clinical Pharmacology 33, 403-407.

Zanni, E. E., Zannis, V. I., Blum, C. B., Herbert, P. N. \& Breslow, J. L. (1987). Effect of egg cholesterol and dietary fats on plasma lipids, lipoproteins, and apoproteins of normal women consuming natural diets. Journal of Lipid Research 28, 518-527. 